\section{Refractory ascites with liver fibrosis developed in late phase allogeneic hematopoietic stem cell transplantation: report of three patients}

\author{
Hiroki Hosoi, ${ }^{1}$ Kenji Warigaya, ${ }^{2}$ \\ Shogo Murata, ${ }^{1}$ Toshiki Mushino, ${ }^{1}$ \\ Kodai Kuriyama, ${ }^{1}$ Akinori Nishikawa, ${ }^{1}$ \\ Shinobu Tamura, ${ }^{1}$ Kazuo Hatanaka, ${ }^{1}$ \\ Nobuyoshi Hanaoka, ${ }^{1}$ \\ Yasuteru Muragaki, ${ }^{3}$ Shinichi Murata, ${ }^{2}$ \\ Hideki Nakakuma, ${ }^{1}$ Takashi Sonoki ${ }^{1}$ \\ 1Department of Hematology and \\ Oncology; 2Department of Human \\ Pathology and Diagnostic Pathology; \\ ${ }^{3}$ First Department of Pathology, \\ Wakayama Medical University, \\ Wakayama, Japan
}

\section{Abstract}

We report cases of three patients of refractory ascites without other fluid retention that occurred around five months after allogeneic hematopoietic stem cell transplantation (alloHSCT). All three patients expired and postmortem examinations revealed unexpected liver fibrosis lacking histological evidences of graft-versus-host-disease (GVHD). The three patients showed normal hepatic function and size before transplantation. During their clinical courses, serum biochemistry test showed no elevation of hepatic enzymes and bilirubin; however, imaging studies demonstrated hepatic atrophy at the onset of ascites. One of the liver specimens showed bile obstruction, which could be seen in hepatic damage by GVHD. Although ascites resulting from venoocclusive disease in early phase allo-HSCT is well documented, ascites associated with hepatic fibrosis in late phase allo-HCST has not been reported. Further clinico-pathological studies on similar patients should be required to ascertain refractory ascites associated with liver fibrosis after allo-HSCT.

\section{Introduction}

Allogeneic hematopoietic stem cell transplantation (allo-HSCT) is an effective therapy for hematological malignancies. This therapy, however, causes fatal complications including opportunistic infections, hepatic veno-occlusive disease and graft-versus-host-diseases (GVHD). As the use of allo-HSCT becomes more widespread, novel complications are still being reported. Therapeutic interventions after the appropriate diagnosis of complications might improve survival after allo-HSCT.

In allo-HSCT, ascites can develop as a symptom of systemic diseases such as cardiac, renal and hepatic insufficiencies. In addition to systemic diseases, infectious peritonitis, sinusoidal obstruction syndrome and GVHDinduced serositis can cause regional ascites. In early phase allo-HSCT, hepatic veno-occlusive disease may cause regional refractory ascites. In late phase allo-HSCT; however, etiologies of regional refractory ascites are little known. We managed three patients who showed refractory ascites without other fluid retention, which occurred at around five months after allo-HSCT. We did not define the origin of the refractory ascites; however, postmortem examinations revealed unexpected liver fibrosis lacking evidences of GVHD. Here, we report the clinical details and histological characteristics of the three patients who showed refractory ascites and liver fibrosis. Representative clinical courses of the three patients are shown in Figure 1.

\section{Case Report \#1}

Patient \#1 was a 62 -year-old male patient with NK/T lymphoblastic lymphoma. This patient showed normal hepatic function based on blood chemistry test before alloHSCT (Table 1). HBs antigen, anti-HBs antibody and anti-HCV antibody were negative for both donor and recipient. This patient received an HLA-matched unrelated bone marrow transplantation after a reducedintensity conditioning regimen at first complete remission. Tacrolimus and methotrexate were used for GVHD prophylaxis. He suffered from human herpes virus-6 (HHV-6) encephalitis on day 20 after HSCT and his condition improved after receiving foscarnet treatment. Five months after HSCT, transudative ascites developed. Considering that the ascites might have been one of the symptoms of chronic GVHD, steroid dose was increased and mycophenolate mofetil was added. Despite immunosuppressive therapy, his ascites deteriorated. At the same time, he suffered from cytomegalovirus colitis, which was treated with foscarnet. Computed tomography (CT) scan revealed liver atrophy (Figure 2A). Abdominal echography also showed liver atrophy, lack of splenomegaly and absence of reversed flow of the portal vein due to portal hypertension. The ascites did not improve despite continuous furosemide infusion. At nine months after HSCT, the ascites exacerbated and the patient expired. Postmortem study revealed that he had $6900 \mathrm{~mL}$ of ascites, liver fibrosis
Correspondence: Takashi Sonoki, Department of Hematology and Oncology, Wakayama Medical University, Kimiidera 811-1, 641-8510 Wakayama, Japan.

E-mail: sonoki@wakayama-med.ac.jp

Key words: Allo-hematopoietic stem cell transplantation; complications; liver fibrosis.

Acknowledgments: this work was supported in part by the Ministry of Education, Culture, Sports, Science, and Technology (MEXT), Japan (No. 25461433) and a Research Grant on Priority Areas from Wakayama Medical University (2012) We would like to thank Mr. Shenli Hew from the Department of Clinical Research Center, Wakayama Medical University, for proofreading and editing the manuscript.

Contributions: HH, SM, TM, KK, AK, ST, KH, NH, HN and TS managed the patients. KW, YM and SM examined the histological characteristics. $\mathrm{HH}$ and TS wrote this manuscript.

Conflict of interest: the authors declare no potential conflict of interest.

Received for publication: 21 February 2016. Accepted for publication: 17 June 2016.

This work is licensed under a Creative Commons Attribution-NonCommercial 4.0 International License (CC BY-NC 4.0).

(C) Copyright H. Hosoi et al., 2016

Licensee PAGEPress, Italy

Hematology Reports 2016; 8:6482

doi:10.4081/hr.2016.6482

and hemochromatosis (Figure 2D,G).

\section{Case Report \#2}

Patient \#2 was a 65 -year-old female patient with an Epstein-Barr virus-positive diffuse large B-cell lymphoma. Her serological tests were positive for anti-HBs and anti-HBc antibodies, and negative for anti-HCV antibody. Hepatic function was normal before allo-HSCT (Table 1). She received a peripheral blood stem cell transplantation from an HLA haplotype-mismatched related donor and a reducedintensity conditioning regimen. GVHD prophylaxis consisted of tacrolimus and methylprednisolone. She showed cytomegalovirus antigenemia on day 10 after HSCT and was treated with foscarnet. As she did not develop acute GVHD, methylprednisolone was tapered rapidly. Six months after HSCT, she developed ascites. CT scan revealed liver atrophy (Figure 2B). Echography also showed liver atrophy and did not detect splenomegaly and cardiac insufficiency. HBV reactivation did not occur 
according to the plasma HBV-DNA level. As the ascites could be one feature of GVHD, we increased steroid dosage. At the same time, EBV reactivation developed, but plasma EBVDNA virus load became negative after rituximab administration. The ascites were not reduced by loop diuretic, aldosterone antagonist and vasopressin receptor antagonist. At 16 months after allo-HSCT, the ascites worsened and the patient expired. Autopsy disclosed that she had $4600 \mathrm{~mL}$ of ascites, liver fibrosis and hemosiderosis (Figure 2E,H).

\section{Case Report \#3}

This patient was a 65 -year-old male with adult T-cell leukemia/ymphoma. His serological tests showed that anti-HBs and anti-HBc antibodies were positive, and that anti-HCV antibody was negative. Blood chemistry test before allo-HSCT indicated normal liver function (Table 1). The patient received bone marrow transplantation from an unrelated HLApartial-mismatched donor after reduced-intensity conditioning chemotherapy at first complete remission. Tacrolimus and methotrexate were used for GVHD prophylaxis. He developed acute skin GVHD (stage III). After steroid therapy, GVHD improved. On day 35 after alloHSCT, cytomegalovirus-antigenemia was positive and foscarnet was administered. Five months after allo-HSCT, the patient suffered from influenza and concurrently developed ascites. He received oseltamivir, peramivir and laninamivir. However, influenza virus antigen was continuously positive in throat swabs for three months. The ascites gradually deteriorated. CT scan revealed liver atrophy (Figure 2C). Polymerase chain reaction analyses were negative for varicella zoster virus, cytomegalovirus, Epstein-Barr virus or tubercle bacillus in ascites. Considering that GVHD might implicate the ascites, we increased the amount of steroid. The ascites did not respond to the continuous infusion of furosemide. The patient expired ten months after allo-HSCT. Postmortem examination revealed that he had suffered from $2500 \mathrm{~mL}$ of ascites, liver fibrosis, cholestasis and liver cirrhosis (Figure 2F,I).

\section{Discussion}

We described three patients showing refractory ascites, which occurred in late phase of allo-HSCT. Autopsies revealed liver fibrosis in the three patients.

Previous studies have reported that persistent hepatic GVHD results in liver fibrosis. ${ }^{1}$ Although the morphological features were not

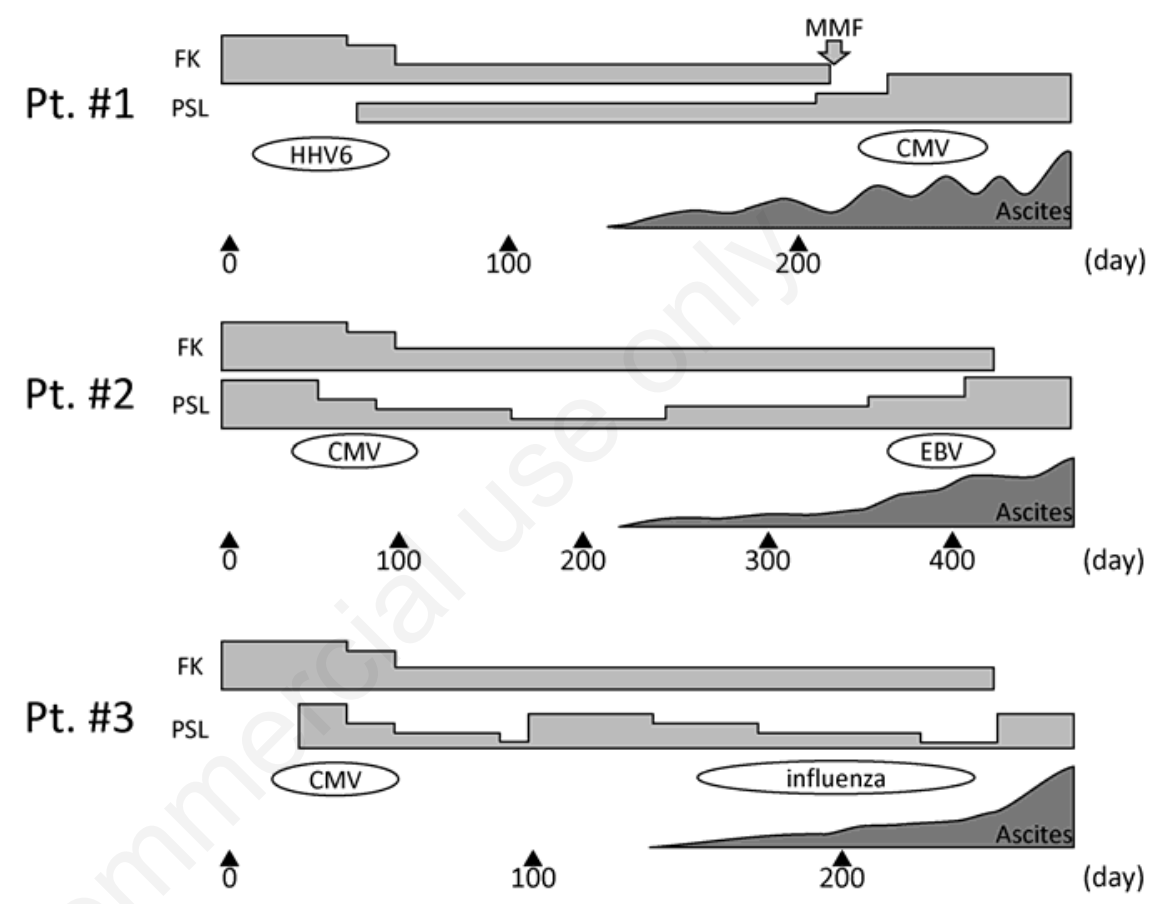

Figure 1. Clinical course of the three patients. Ascites developed beyond 100 days after allogeneic hematopoietic stem cell transplantation. All patients suffered from multiple viral infections.

Table 1. Laboratory findings of the three patients.

\begin{tabular}{|c|c|c|c|c|c|c|}
\hline & \multicolumn{2}{|c|}{ Patient \#1 } & \multicolumn{2}{|c|}{ Patient \#2 } & \multicolumn{2}{|c|}{ Patient \#3 } \\
\hline & Before HSCT & At ascites & Before HSCT & At ascites & Before HSCT & At ascites \\
\hline PT-INR & 1.02 & 1.0 & 1.1 & 0.4 & 0.92 & 1.1 \\
\hline T-Bil (mg/dL) & 0.4 & 0.5 & 0.4 & 0.3 & 0.5 & 2.3 \\
\hline D-Bil (mg/dL) & 0.0 & 0.0 & 0.0 & 0.0 & 0.1 & 1.1 \\
\hline Alb (g/dL) & 4.1 & 2.9 & 3.3 & 3.1 & 4.8 & 3.3 \\
\hline ChE (U/L) & 199 & 154 & 156 & 175 & 360 & 136 \\
\hline AST (U/L) & 26 & 18 & 12 & 35 & 29 & 51 \\
\hline $\operatorname{ALT}(\mathrm{U} / \mathrm{L})$ & 16 & 15 & 5 & 29 & 19 & 28 \\
\hline ALP (U/L) & 285 & 305 & 318 & 569 & 237 & 282 \\
\hline GTP (U/L) & 151 & 75 & 37 & 269 & 24 & 131 \\
\hline HBs ag & - & - & - & - & - & NT \\
\hline anti-HBs ab & - & - & + & + & + & NT \\
\hline anti-HBc ab & - & - & + & NT & + & NT \\
\hline anti-HCV ab & - & - & - & - & - & NT \\
\hline
\end{tabular}

NT, not tested. 
Pt. \#1
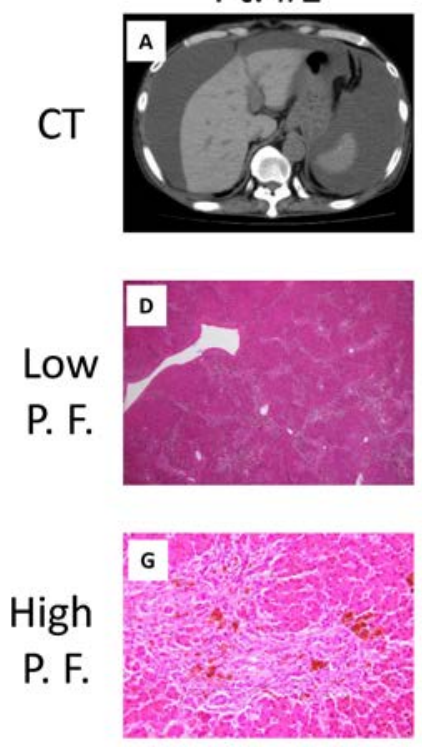

Pt. \#2
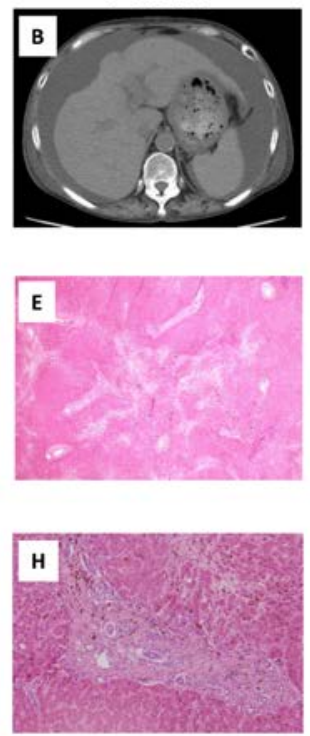

Pt. \#3
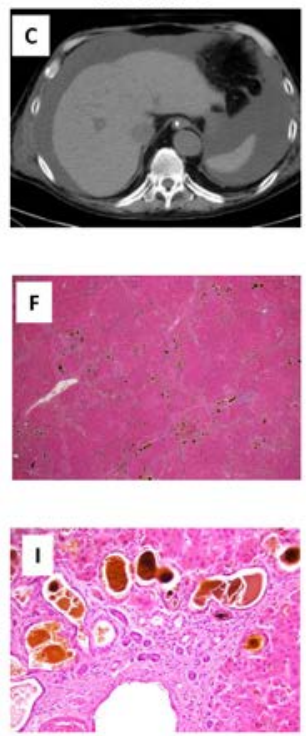

Figure 2. Computed tomography (CT) and pathological findings. A-C) CT images of the ascites and liver. D-F) Microscopic findings of the liver specimens at low-power field $(20 \times)$. All specimens showed fibrosis in portal spaces. G-I) High-power field images (G,H: x200, I: 100x). Lymphocyte infiltration was not seen in all specimen. In patient 3 , bile plug due to cholestasis was seen (I). A, D and G) patient no. 1; B, E and H) patient 2; C, F and I) patient no. 3

consistent with typical hepatic GVHD, intensive immunosuppressive therapy might alter the histological characteristics. Therefore, we could not rule out the possible involvement of GVHD in the liver fibrosis. In view of possible involvement of GVHD, patient no. 3 showed cholestasis, one of the histological features in chronic hepatic GVHD. ${ }^{2}$ In addition to hepatic GVHD, other pathological conditions could also lead to the development of liver fibrosis. For example, chronic viral hepatitis induces liver fibrosis. Liver fibrosis caused by hepatitis virus progresses slowly over 20 years leading up to liver cirrhosis. ${ }^{3,4}$ However, liver fibrosis in the three patients advanced rapidly within half a year after allo-HSCT. Liver fibrosis after allo-HSCT advanced more rapidly than viral hepatitis. In addition, liver fibrosis after transplantation brought refractory ascites drastically. Thus, it is important to take note of the signs for liver fibrosis after alloHSCT. Although the three patients suffered from liver fibrosis, their serum liver enzymes were not elevated. For that reason, we did not notice the liver fibrosis. Biomarkers of chronic liver GVHD such as serum bilirubin and alkaline phosphatase were also unchanged. ${ }^{5}$ Instead of liver enzymes, patient no. 2 showed elevated biomarkers of fibrosis such as hyaluronic acid and type IV collagen. The fibrotic biomarkers of the other two patients were not measured. In addition, all three patients exhibited liver atrophy on CT analyses. Thus, biomarkers of fibrosis and atrophic change of the liver may be clues to identify liver fibrosis. All three patients suffered from multiple viral infections. A previous report suggested that effusions as part of the presentation of GVHD were often associated with cytomegalovirus disease. ${ }^{6}$ Our patients had persistent viral infection before the onset of ascites. These infections might enhance immune reaction and trigger liver fibrosis.

Multiple infections and refractory viral infections may be associated with the onset of liver fibrosis carrying refractory ascites.

\section{Conclusions}

We report three patients showing refractory ascites with liver fibrosis that developed in late phase allo-HSCT. All patients' conditions deteriorated and fatal clinical courses. Thus, accumulation of similar cases and further studies are required for appropriate therapeutic interventions for such uncontrollable complication of allo-HSCT.

\section{References}

1. Shulman HM, Sharma P, Amos D, et al. A coded histologic study of hepatic graft-versus-host disease after human bone marrow transplantation. Hepatology 1988;8: 463-70.

2. Shulman HM, Cardona DM, Greenson JK, et al. NIH Consensus development project on criteria for clinical trials in chronic graft-versus-host disease: II. The 2014 Pathology Working Group Report. Biol Blood Marrow Transplant 2015;21: 589-603.

3. Poynard T, Bedossa P, Opolon P. Natural history of liver fibrosis progression in patients with chronic hepatitis $\mathrm{C}$. The OBSVIRC, METAVIR, CLINIVIR, and DOSVIRC groups. Lancet 1997;349:825-32.

4. Peffault de Latour R, Levy V, Asselah T, et al. Long-term outcome of hepatitis $\mathrm{C}$ infection after bone marrow transplantation. Blood 2004;103:1618-24.

5. Greinix HT, Loddenkemper C, Pavletic SZ, et al. Diagnosis and staging of chronic graft-versus-host disease in the clinical practice. Biol Blood Marrow Transplant 2011;17:167-75.

6. Seber A, Khan SP, Kersey JH. Unexplained effusions: association with allogeneic bone marrow transplantation and acute or chronic graft-versus-host disease. Bone Marrow Transplant 1996;17:207-11. 